\section{PS-030 CONGENITAL HEART DISEASE AND MICROCEPHALY AT BIRTH: A DANISH POPULATION-BASED COHORT STUDY OF CHILDREN WITH DOWN SYNDROME}

${ }^{1}$ NB Matthiesen, ${ }^{2} \mathrm{JW}$ Gaynor, ${ }^{1}$ TB Henriksen, ${ }^{3}$ VE Hjortdal, ${ }^{1} \mathrm{P}$ Agergaard, ${ }^{1} \mathrm{JR}$ Ostergaard. ${ }^{1}$ Department of Pediatrics, Aarhus University Hospital, Aarhus, Denmark; ${ }^{2}$ Division of Cardiothoracic Surgery, The Children's Hospital of Philadelphia, Philadelphia, USA; ${ }^{3}$ Department of Cardiothoracic Surgery, Aarhus University Hospital, Aarhus, Denmark

\subsection{6/archdischild-2014-307384.325}

Background and aims Neurodevelopmental disorders are common in children with congenital heart disease (CHD) and largely ascribed to prenatal factors such as impaired cerebral growth. It remains to be established whether this is due to impaired intrauterine cerebral blood flow or genuine genetic causes. Down syndrome (DS) is a known cause of CHD, neurodevelopmental disorders and microcephaly. Hence, studies on DS may provide insight into the causes of impaired cerebral growth in CHD. We aimed to assess the risk of microcephaly in children with DS and CHD compared to children with DS and no CHD.

Methods Children with DS $(\mathrm{n}=389)$ and specific birth characteristics were identified in national registries. Head circumference and the risk of microcephaly (head circumference <-2SD) was compared between children with CHD $(\mathrm{n}=168)$ and children without CHD $(n=221)$ by linear and logistic regression analyses (unadjusted and adjusted for gender and gestational age).

Results There was no difference in head circumference between the groups, $0.0 \mathrm{~cm}$ (95\% CI -0.4-0.4). Adjustment did not significantly alter the results. The risk of microcephaly was slightly higher in newborns with CHD, OR 1.4 (95\% CI 0.8-2.6). Adjustment did not significantly alter the results.

Conclusions We did not find indications of impaired head growth in children with DS and concomitant CHD. There might be a slight increase in the risk of microcephaly. We suggest that the most common types of CHD in DS i.e. atrioventricular septal defects, ventricular septal defects and atrial septal defects do not impair prenatal cerebral growth in children with DS.

\section{PS-031 CONGENITAL HEART DISEASE IN THE IN-VITRO FERTILISATION PREVALENCE DISTRIBUTION}

${ }^{1}$ F Chen, ${ }^{2} \mathrm{M}$ Lee. 'Department of Pediatrics, Chung Shan Medical University Hospital, Taichung, Taiwan; ${ }^{2}$ Department of Obstetrics and Gynecology, Chung Shan Medical University Hospital, Taichung, Taiwan

\subsection{6/archdischild-2014-307384.326}

Introduction A higher prevalence of all congenital malformations in children conceived through assisted reproductive technologies (ART) was fairly extensively suggested in literature. However, there are few studies which only address congenital heart disease (CHD) specifically and most have examined data from registers. The aim of this study was determined the prevalence of CHD in fetus and newborn conceived by ART and comparison with newborn in outpatient obstetric clinics. All data was collected in a specialist paediatric service in the Taichung Taiwan.

Methods This study was detected fetus and newborns with CHD. All pregnancies treated by ART who received fetal echocardiography between gestational age from 20 to 24 weeks and examine done month after delivery or reported by echocardiographic screening from their birth hospital. The controlled group was normal conceived newborn receiving postnatal echocardiographic screening at age of one week at the same area in Taichung city.

Results Among 2,780 fetus in ART, the prevalence of major CHD with immediate life-threatening risk and overall CHD were 503.5/100,000 $(\mathrm{n}=14)$ and 1,223/100,000 $(\mathrm{n}=$ 34), respectively. In comparison with control group of 12,022 newborn, the major CHD was 2 times of normal pregnancy but all CHD were only one half of normal pregnancy. In assisted conception were observed increased in a number of individual subgroups rates, the highest among those with pulmonary atresia.

Conclusions We found no increased risk of overall CHD but more risky in major CHD in those conceived by ARTs. Further analysis of individual subgroups of CHD and different methods of conception is required. Prenatal echocardiographic screening can play a useful tool for early detection of major CHD in ART to improve the outcome of CHD.

\section{PS-032 PERFUSION INDEX IN PRETERM INFANTS DURING THE FIRST 3 DAYS OF LIFE}

T Alderliesten, PMA Lemmers, F van Bel. Neonatology, Wilhelmina Children's Hospital University Medical Center Utrecht, Utrecht, Netherlands

\subsection{6/archdischild-2014-307384.327}

Background and aim The waveform amplitude produced by pulse oximeters can be expressed as an index of pulsatile vs. non-pulsatile signal. This perfusion index (PI) has been shown to correlate with cardiac output, stroke volume, and superior vena cava flow. The aim was to gather PI reference data in preterm infants and to explore if the PI is associated with common clinical parameters.

Patients/methods The PI was recorded in 312 neonates $<32$ weeks GA during the first $72 \mathrm{~h}$ of life. Mixed-effects modelling was applied with PI as the dependent variable and the individual patient as a random factor. Subsequently the association with clinical parameters (i.e. GA, birth weight, IVH, PDA, inotropes) was explored.

Results Mean GA was 28.5 weeks (SD \pm 2.1$)$. A quadratic model $(0-24 \mathrm{~h})$ combined with a linear model $(24-72 \mathrm{~h})$ provided the best fit. The lowest PI was reached 12-18 h after birth, thereafter gradually increasing until $72 \mathrm{~h}$ postnatal age. For the first $24 \mathrm{~h}$ PI was associated with gender (coefficient $0.05, \mathrm{p}=0.04)$, inotrope administration $(-0.123, \mathrm{p}<0.0001)$, pulse pressure $(0.014, \mathrm{p}<0.0001), \mathrm{SaO}_{2}(-0.015, \mathrm{p}<0.0001)$, MABP $(-0.013, p<0.0001)$, and GA $(0.014, p=0.0168)$. After the first day, only associations with, inotrope administration $(-0.17, \mathrm{p}<0.0001)$, pulse pressure $(0.007, \mathrm{p}<0.0001)$, MABP $(-0.014, \mathrm{p}<0.0001)$, and $\mathrm{SaO}_{2}(-0.01, \mathrm{p}<0.0001)$ remained. No association was found with, IVH, PDA, fluid boluses, or birth weight.

Conclusions The evolution of PI values over time probably reflects transitional physiology. The associations with pulspressure, MABP, and inotrope administration suggest that the PI might have an application in blood pressure management. 